\title{
Variations
}

Variations

Revue internationale de théorie critique

$23 \mid 2020$

Pour une gauche érotique

\section{Critique de l'économie politique et industrie culturelle}

Généalogie du marxisme adornien

Christophe Magis

\section{(2) OpenEdition}

Journals

Édition électronique

URL : https://journals.openedition.org/variations/1703

DOI : $10.4000 /$ variations. 1703

ISSN : 1968-3960

Éditeur

Les amis de Variations

Référence électronique

Christophe Magis, "Critique de l'économie politique et industrie culturelle », Variations [En ligne], 23 |

2020, mis en ligne le 01 septembre 2020, consulté le 21 septembre 2021. URL : http://

journals.openedition.org/variations/1703 ; DOI : https://doi.org/10.4000/variations.1703

Ce document a été généré automatiquement le 21 septembre 2021.

Les ami•e•s de Variations 


\section{Critique de l'économie politique et industrie culturelle}

Généalogie du marxisme adornien

\section{Christophe Magis}

1 Le chapitre central de La dialectique de la raison s'ouvre sur un premier paragraphe de réflexion générale à propos du modèle de la «civilisation de masse ». Le terme allemand Kultur possède en effet une acception plus large que ce qu'en français on rend par "culture ", pour englober également les faits de civilisation. Il n'est donc pas si étonnant qu'un chapitre titré «Kulturindustrie» commence par poser les traits civilisationnels saillants de l'époque qu'il analyse. En fait, ces traits convergent ici en un seul, la similarité: "La civilisation actuelle confère à tout un air de ressemblance [ Ähnlichkeit] $»^{1}$. Et ce trait est immédiatement rapproché d'une cause économique sousjacente à toute civilisation de masse : la puissance qu'y exercent les monopoles. Afin de saisir de manière adéquate le cœur de ce qui est critiqué dans ce chapitre, il convient dans un premier temps de rappeler la critique économique et sociale dans laquelle les « fragments philosophiques » s'insèrent.

2 Quoiqu'assez rarement exploré, le sujet n'est pas tout à fait vierge. Plusieurs commentateurs ont indiqué combien la pensée d'Horkheimer et Adorno avait été influencée par un ensemble de débats économiques et politiques qui occupaient l'Institut de recherches sociales au moment de la rédaction de leur ouvrage : la querelle $\mathrm{du}$ «capitalisme d'État $»^{2}$. Ces débats sont au cœur du déplacement, opéré par la Théorie critique des années 1940, par rapport au marxisme traditionnel qui avait pu baigner un certain nombre de travaux précédents : ils questionnent la prééminence de la détermination économique au profit de la détermination politique dans le capitalisme d'après sa période libérale. Certains travaux ont également montré combien, tandis que la première version de La dialectique de la raison, inédite, n'a circulé dès 1944 qu'au sein du cercle restreint de l'Institut für Sozialforschung émigré à NewYork, la publication effective de l'ouvrage en 1947 a introduit un certain nombre de modifications terminologiques, notamment pour ce qui concerne le chapitre sur la «production industrielle de biens culturels ${ }^{34}$. Ces modifications concernent 
principalement des expressions économiques, et justement, très majoritairement cette catégorie de «monopole ». Ainsi, comme le montrent Willem van Reijen et Jan Bransen, si l'on compare les deux textes :

"Le terme "monopole" devient "l'appareil économique" ou est remplacé par "les agences de production de masse", "le système de l'industrie moderne", "l'industrie culturelle", "ceux qui les organisent [les masses]", ou de manière plus neutre, "le système". Il peut également être remplacé par "les trusts" et "cartels". Les "dirigeants des monopoles" sont transformés en "directeurs généraux". Le "capital" devient "l'économie", son "pouvoir" devient "le pouvoir de ceux qui dominent économiquement". Il arrive par endroits au "capitalisme" de disparaître complètement. $»^{5}$

3 Pour van Reijen et Bransen, de tels changements ne peuvent s'expliquer, comme on l'avance quelquefois, uniquement par la simple nécessité, pour Horkheimer et Adorno, de ne pas trop "se faire remarquer » en tant qu'intellectuels marxistes étrangers ${ }^{6}$, et révèlent plutôt combien l'édition de 1947, après un ensemble de travaux sur les mutations de l'économie capitaliste et de la structure de la lutte des classes, s'éloigne d'une forme de marxisme selon laquelle la dimension économique représentait le facteur déterminant. L'industrie culturelle serait alors la pièce maîtresse d'une nouvelle conception de la domination, d'ordre définitivement politique.

4 Le présent article invite à une position plus dialectique. Quoique, et notamment dans l'ouvrage écrit à quatre mains, la dimension politique soit fondamentale pour le concept d'industrie culturelle, ce dernier n'en représente pas moins un concept pivot permettant d'amorcer une forme particulière de critique de l'économie politique et de ses catégories vers la description d'une société dans laquelle la domination tend de plus en plus à être exercée par des abstractions.

$5 \quad$ Il s'agit donc d'explorer les rapports du concept d'industrie culturelle aux mutations du capitalisme telles que les réfléchissent les auteurs de l'École de Francfort. Il est fréquemment rapporté que Horkheimer et Adorno n'ont jamais été de bons élèves en ce qui concerne l'économie politique'. Et il faut reconnaître que leurs intérêts théoriques comme les travaux qu'ils ont produits visent davantage la réflexion philosophique, sociologique, psychologique et esthétique. Mais il n'en demeure pourtant pas moins qu'on ne peut saisir sérieusement l'industrie culturelle telle qu'elle est entendue par les deux auteurs sans la rapporter aux évolutions de leur pensée économique et des milieux intellectuels dans lesquels ils ont baigné. Nous proposons donc de restituer les débats économiques ayant divisé, au début des années 1940, les membres de l'Institut de recherches sociales reconstitué à New-York, dans un premier temps, afin de montrer combien l'industrie culturelle, telle que conceptualisée dans La dialectique de la raison, s'appuie sur le développement de la notion de capitalisme d'État et surtout de sa réception par Horkheimer avec sa catégorie d'«État autoritaire ». Nous suivrons enfin le concept au fur et à mesure des développements ultérieurs d'Adorno, jusqu'aux derniers textes, autour de la catégorie de capitalisme tardif.

\section{Débats de l'Institut de recherches sociales, entre économie et politique}

Si ni Horkheimer, ni Adorno ne sont des spécialistes de l'économie, cela n'empêche que le début des années 1940 est largement consacré, pour l'ensemble des penseurs de l'Institut de recherches sociales exilé à New-York, à des analyses économiques et 
politiques. L'enjeu est d'analyser les conditions d'émergence de l'État totalitaire national-socialiste qu'ils ont fui. Et dans ce cadre, la figure de Friedrich Pollock - à qui La dialectique de la raison est d'ailleurs dédiée - est primordiale, tant pour le caractère novateur de sa position que pour son influence intellectuelle sur les principaux penseurs de l'École de Francfort comme Horkheimer, Marcuse et Adorno. Économiste de formation, Pollock avait entamé dès les années 1930 une analyse du développement $\mathrm{du}$ capitalisme sortant des propositions économiques marxistes d'inspiration plus "classique ", formulées par des membres issus de la première génération de l'Institut, avant la prise de direction par Horkheimer, notamment celles de Henryk Grossmann ${ }^{8}$.

7 Pollock montrait ainsi combien la Grande Dépression, loin d'être le fossoyeur du capitalisme, était la crise de sa seule phase libérale, celle s'appuyant sur le marché comme instance de régulation des entreprises privées. L'époque était mûre pour une nouvelle phase de développement, celle de la planification de l'économie par l'État interventionniste. Cette hypothèse a été synthétisée dans l'essai de 1941 «State Capitalism: It's Possibilities and Limitations" dans lequel Pollock définit cette nouvelle phase du capitalisme planifié par ce qui la différencie de manière fondamentale de la phase libérale: le marché n'est plus l'instance de contrôle et de coordination de la production et de la distribution (et les «lois de l'économie " disparaissent avec lui); c'est à l'État que ce contrôle est dévolu (qui peut recréer une forme de "pseudomarché »)9. Il existe deux formes idéal-typiques d'un tel « capitalisme d'État » : la forme totalitaire (qui inclut à la fois l'État nazi et l'État soviétique), où l'« État est instrument de pouvoir d'un nouveau groupe dirigeant " qui comprend les patrons d'industrie, les hauts fonctionnaires de la bureaucratie d'État et les cadres du parti; et la forme démocratique, où l'appareil d'état est contrôlé par le peuple (cette forme étant plus difficile à exemplifier). Dans les deux cas, la résultante en est un ascendant du politique sur l'économique ainsi qu'un décentrement de la contradiction fondamentale du capitalisme depuis la sphère de la production (i.e. la tension entre forces productives et rapports de production qui caractérise le mode de production capitaliste fondamentale pour la critique marxiste classique) vers celle de la distribution et sa régulation. Ainsi, pour Pollock, l'état national-socialiste est le signe paradigmatique d'un "nouvel ordre $»^{10}$, caractérisé par le fait que "la primauté du politique sur l'économique, si controversée en démocratie, [y] est clairement établie ${ }^{11}$. Et corollairement, c'est davantage la recherche de pouvoir au sein de l'appareil politique qui tend à devenir la pulsion motrice, plutôt que la recherche de profit, plus typique de la période précédente.

8 Il en découle un certain nombre de "nouvelles règles du jeu» dans lesquelles la direction de la production, les prix et les profits s'articulent autour de "plans" d'ensemble. La gestion en est assurée selon « les principes du scientific management ", en remplacement du tâtonnement confus et désorganisé qui organisait, via le marché, la phase précédente ${ }^{12}$. L'économiste, qui devait autrefois «se creuser la cervelle afin de résoudre le casse-tête du procès d'échange ", n'a affaire, dans le capitalisme d'État, qu'à « des problèmes d'ordre administratif $»^{13}$. Et la fonction d'investisseur se sépare, dans les entreprises, de celle de la direction, qui devient « indépendante » du capital investi. Ainsi, « le capitaliste - s'il n'est pas reconnu comme entrepreneur sur la base de ses qualités gestionnaires - est transformé en simple rentier ${ }^{14}$. Cette thèse rappelle celle de l'Ère des organisateurs, de James Burham, plus connue aux États-Unis par la suite. La structuration hiérarchique de la société totalitaire préfigure une «révolution 
managériale » amenée à s'étendre aux autres États et dans laquelle une nouvelle classe, celle des organisateurs est amenée à prendre la direction de la société et ce, indépendamment de la condition économique de ses membres.

Les conséquences sociales de ces transformations sont importantes. Dans la «société traditionnelle", qui comprend, pour Pollock, le capitalisme dans sa phase libérale, «individus et classes communiquent à travers le médium de l'échange, en tant qu'agents légalement égaux $»^{15}$. Quoique cette situation légale ait historiquement, en réalité et derrière l'apparence, constamment dissimulé le caractère inégal des rapports sociaux et la violence de la domination, elle assurait néanmoins une autonomie des individus. La constitution du sujet bourgeois s'appuyait sur le caractère extérieur d'un domaine juridique ainsi défini, qui assurait la séparation de la sphère publique et de la sphère privée. De son côté, le capitalisme d'État a aboli la notion même d'acteurs économiques autonomes et, partant, l'indépendance de l'individu ${ }^{16}$. Toutes les sphères de la vie sociale devenant domaines d'activité de l'État, elles se structurent selon le modèle hiérarchisé de la bureaucratie politique : « les hommes se rencontrent en tant que commandant et commandé $\aleph^{17}$, soumis à une rationalité technique unilatérale. Cette rationalité, qui transforme l'appareil d'État en machine de très haute précision technique, exige une efficacité tout aussi mécanique de la part de l'individu, qui est totalement subordonné au tout. Sa satisfaction privée est par ailleurs devenue affaire publique : au prix de la perte de son autonomie individuelle, le sujet bénéficie d'un desserrement relatif de certaines normes sociales - que le capitalisme d'État réoriente par ailleurs en direction de ses plans : par exemple, dans le cadre de l'État NationalSocialiste, l'allègement des tabous sexuels s'accompagne de politiques nataliste et la libération des instincts est détournée vers une haine des ennemis du régime (Juifs, faibles d'esprit, personnes inaptes) ${ }^{18}$.

Mais si la thèse du capitalisme d'État présuppose une intégration politique progressive de toutes les oppositions, sa réception n'a pas été sans contestation. Rapidement, Franz Neumann, un autre membre de l'Institut, juriste formé à la London School of Economics, propose une critique du concept de "capitalisme d'État» et de son affirmation essentielle quant au déplacement de l'instance de régulation depuis l'économique vers le politique, notamment dans le cadre de l'analyse de l'État national-socialiste. Pour Neumann, quoique la thèse de Pollock soit « un point de vue séduisant, parce qu'il fait ressortir la différence entre le national-socialisme et la démocratie, sous un angle non seulement politique et idéologique, mais aussi économique $»^{19}$, elle n'est pas vraiment tenable ni théoriquement, ni empiriquement. Au point de vue théorique, «la formule même de "capitalisme d'État" est une contradiction dans les termes $"^{20}$ : si l'État était propriétaire unique il ne pourrait y avoir à proprement parler d'économie capitaliste on serait sorti du capitalisme. C'est ce qui, selon l'auteur, permet à la thèse de Pollock de négliger les contradictions internes au système et d'en postuler à la fois la stabilité et le maintien dans la durée. Ce pessimisme est, pour Neumann, dû notamment à un biais méthodologique: la catégorie de capitalisme d'État est affirmée comme un idéaltype mais élaboré selon une méthode qui ne la déduit pas de la réalité. Dès lors que " ses défenseurs décrivent un système complètement étranger au capitalisme, qui est en fait son contraire absolu, et qui implique un saut qualitatif d'une réalité à l'autre ", il serait nécessaire, pour valider une telle thèse, de «montrer avec précision en quoi le capitalisme a cessé d'exister en Allemagne. $»^{21}$ 
$11 \mathrm{Au}$ contraire, par l'étude effective, minutieuse et s'appuyant sur de nombreuses données empiriques de l'État national-socialiste, Neumann démontre combien celui-ci, quoique visant une intégration politique la plus totale possible, ne résout pas tant les contradictions économiques du capitalisme libéral qu'il les exacerbe. Dans les nombreux éléments empiriques qu'il rapporte, Neumann révèle combien les bénéficiaires véritables des nouveaux trusts, sous l'État national-socialiste, sont bien plutôt les anciennes dynasties entrepreneuriales qu'une véritable « nouvelle classe » de managers. Fidèle à un marxisme plus traditionnel, l'auteur montre ainsi que c'est toujours «la recherche du profit qui maintient la cohésion de toute la machine économique $»^{22}$.

Bien sûr, cela ne signifie pas que l'État nazi ne présente pas de différence politique ou économique spécifique. Neumann intègre en réalité la proposition pollockienne de l'ascendant progressif du politique mais en l'intégrant à une analyse des mutations de l'économie capitaliste depuis sa phase libérale. Pour lui, il convient d'analyser le national-socialisme à partir du modèle du "capitalisme monopoliste ». Si le contrat a été, pendant la phase libérale, le concept juridique garant de la propriété, de la libre concurrence et, partant, de la liberté du commerce, il a peu a peu été hypostasié en absolu juridique et séparé du contexte socio-économique. Il a alors permis la formation de coalitions industrielles et «la liberté du commerce a dégénéré au point de n'être plus qu'une pure formule de propagande pour la défense des prérogatives économiques ${ }^{23}$ et des privilèges. C'est l'établissement des monopoles industriels. Mais en se maintenant, la liberté de contrat, qui autorise la constitution des syndicats opposant le pouvoir collectif des travailleurs à celui des trusts, représente aussi une arme contre les monopoles, qui permet de résister aux pressions à la réduction des salaires et au licenciement, rendant les périodes de dépression très inconfortables pour les monopoles. « Pour les deux camps - d'un côté la grande masse de la population et le petit entrepreneur, de l'autre les puissances monopolistes - , l'intervention étatique devient dès lors le problème essentiel »: les uns demandent la protection étatique, les autres l'abrogation de la liberté de contrat. «La question la plus importante de la société moderne est de savoir qui doit intervenir, et en faveur de qui. La possession de l'appareil d'État est alors l'axe autour duquel tout le reste s'organise. C'est seulement en ce sens qu'on peut parler d'une primauté du politique sur l'économique. " ${ }^{24}$ Pour Neumann, c'est en tant qu'aide au profit privé et à la préservation des gains des monopoles que le pouvoir politique totalitaire a été mis en place. Le national-socialisme représente donc plutôt un prolongement de l'économie précédente, dans lequel l'État a pour fonction de « supprimer et d'éliminer la liberté politique et économique au moyen des nouvelles garanties auxiliaires de la propriété - le dirigisme, l'intervention de l'administration -, faisant ainsi entrer de force l'ensemble de l'activité économique allemande dans le réseau des ententes industrielles dirigées par les magnats de l'industrie ${ }^{25}$. Partant, c'est « une économie capitaliste privée encadrée par l'État totalitaire », un "Capitalisme Monopoliste Totalitaire ${ }^{26}$. Il s'agit donc bien d'une organisation politique nouvelle, mais qui émerge toutefois en réponse à un problème posé par les contradictions de la structure économique et la difficulté pour les institutions démocratiques de satisfaire les revendications des monopoles, que les mutations du capitalisme ont constitué. C'est un enrégimentement de l'État au service du capitalisme tel qu'il existe, condition essentielle de la coopération industrielle :

«Le national-socialisme recherche la gloire et la consolidation de sa domination;

l'industrie recherche la pleine utilisation de ses capacités et la conquête de marchés 
extérieurs. [...] Le national-socialisme s'est servi de l'audace, de la compétence, de l'agressivité de l'industrie, qui de son côté s'est servir de l'anti-démocratisme, de l'anti-libéralisme et de l'anti-syndicalisme du parti national-socialisme. ${ }^{27}$

\section{De l'« État autoritaire » à l'« industrie culturelle »}

13 Si Adorno, qui n'a rejoint l'Institut de manière permanente qu'en 1938, n'a participé au débat sur le capitalisme d'État que de manière périphérique ${ }^{28}$, il n'en demeure pas moins que l'analyse de l'industrie culturelle, qu'il proposera dans l'ouvrage écrit avec Horkheimer, n'est pas sans porter un certain nombre de marques de cette grande réflexion sur les mutations du capitalisme.

À la manière d'autres membres de l'Institut, Horkheimer a, de son côté, globalement fait sienne l'hypothèse du capitalisme d'État à partir des années 1940, quoique d'une manière singulière. L'étude sur l'« État autoritaire », publiée en 1942, affirme ainsi que «le capitalisme d'État est l'État autoritaire du temps présent ${ }^{29}$. Horkheimer s'y propose de mettre en lumière les transformations économiques, sociales et politiques survenues depuis la Révolution bourgeoise jusqu'aux États nazi et stalinien. L'analyse se place globalement dans le cadre théorique de Pollock, et adhère à la thèse d'une mutation fondamentale du capitalisme, caractérisée par l'ascendant du politique sur l'économique: «L'économie adéquatement planifiée peut mieux nourrir la masse et mieux se laisser nourrir par elle que par les restes du marché. Une période avec sa structure sociale propre a pris la place de la libre économie $\star^{30}$. Il s'agit notamment de réfléchir cette nouvelle période en pointant combien le dépassement des contradictions du libéralisme n'y produit pas l'émancipation mais au contraire une plus grande répression et une plus grande domination. Ces dernières, qui étaient en germe dans l'État libéral, sont désormais libérées.

Pour Horkheimer, «[1]a forme la plus cohérente d'État autoritaire [...] est l'étatisme intégral ou socialisme d'État » qui ne représente pas un recul quant à la recherche de productivité mais au contraire « intensifie la production comme seul l'a fait le passage de la période mercantiliste à la période libérale $»^{31}$. Les États fascistes, de leur côté, sont une "forme mixte» car, si la plus-value y «est gagnée et répartie sous contrôle étatique", "elle continue cependant, sous l'ancien titre de profit, de s'écouler abondamment vers les magnats de l'industrie et les propriétaires fonciers $\aleph^{32}$. On voit ici combien Horkheimer vise à dépasser certaines contradictions du débat sur le capitalisme d'État. Quelle qu'en soit la forme, l'État autoritaire est toujours répressif et il organise une domination de type bureaucratique qui n'équivaut en aucun cas, même pour l'étatisme intégral, à une socialisation véritable mais plutôt à une " parodie de la société sans classes ${ }^{33}$, visant à voiler la violence des antagonismes sociaux qui continuent de la structurer. Ainsi le philosophe, comme ses collègues de l'Institut, affirment-ils leur scepticisme radical quant à la prétention du soviétisme d'avoir effectivement dépassé le capitalisme et, partant, au marxisme traditionnel, tel qu'il s'incarne dans les organisations partisanes ou syndicales, de permettre ce dépassement. Horkheimer va également ici au-delà de la position de Pollock pour qui le capitalisme d'État, en tant que forme qu'il voit la plus en phase avec le développement des forces historiques, pourrait surmonter la crise vers l'établissement de sa variante " démocratique ». 
Toutefois, l'État autoritaire ne définit pas le modèle d'un type de régime totalitaire ni ne vise à saisir véritablement la spécificité de la domination dans le cadre d'un tel régime. Dans son étude sur Horkheimer, Katia Genel analyse ainsi combien cette indétermination entre autoritarisme et totalitarisme permet au philosophe de " figurer des tendances fascistes contre la démocratie et des tendances fascistes dans la démocratie $»^{34}$. En réalité, au-delà des totalitarismes pointés et bien au-delà des stricts problèmes d'économie politique, la catégorie de l'État autoritaire permet de désigner, pour Horkheimer, le processus général de la bureaucratisation, qui est critiqué en tant que devenir autoritaire de l'idéal bourgeois, en germe dans les différentes formes d'État depuis la Révolution française où le Comité de salut public était déjà une première forme centralisée dans laquelle l'« État réglementait l'économie ». Même la mise en place de l'économie de marché n'a pu éradiquer tout à fait la tendance et « la croissance de la puissance capitaliste ne put plus, par la suite, coïncider avec les intérêts généraux que dans le monde des économistes $»^{35}$. Certes, le caractère irrationnel de l'appropriation privée a été critiqué par d'authentiques progressistes au sein des unions de travailleurs. La nécessité, défendue par ces dernières, de dépasser la propriété privée des moyens de production, celle d'une organisation plus rigoureuse de l'économie au moyen de la planification, afin de réduire le gaspillage "étaient des revendications rationnelles à l'époque ». Mais il semble que la question de la liberté individuelle, de sa conquête, de son maintien et de sa garantie, ait été reléguée à l'état de conséquence, de "suite logique, mécanique, de la prise de pouvoir». L'État autoritaire montre qu'il n'est pas de telle conséquence mécanique : le dépassement du libéralisme (première phase du capitalisme) a pris la direction de la planification, avec la formation des monopoles, mais s'est accompagné d'un accroissement de la répression. Horkheimer accuse d'utopie la position, partagée par de nombreux marxistes depuis Engels, selon laquelle la socialisation signifierait la fin de la domination: "chaque fois qu'une étape de la planification serait accomplie, une part de répression devait devenir superflue. En fait, la répression s'est toujours davantage cristallisée, dans le contrôle des plans $»^{36}$.

17 Ainsi, Horkheimer critique-t-il le devenir totalitaire de la planification engendrant, dans les États totalitaires autant que dans les pays démocratiques, une domination bureaucratisée qui s'appuie sur un ressort anthropologique simple: celui de l' adaptation, "prix qu'individus et associations doivent payer pour s'épanouir dans le capitalisme ». Et l'adaptation concerne aussi les forces critiques qui ont eu un rôle important dans la mise en place de la planification. Les mutations décrites ont donc également des conséquences profondes sur la critique sociale. L'article s'appesantit notamment sur la transformation des organisations de travailleurs :

"Le mot d'ordre d'union dans les syndicats et dans les partis fut observé à fond, mais ceux-ci s'occupèrent moins de mener à bien les tâches [...] des prolétaires unis, à savoir l'opposition à la société de classes en général, qu'ils n'obéirent aux conditions naturelles de leur propre développement, les menant à une organisation de masses. ${ }^{37}$

18 Syndicats et partis tendent à fonctionner en miroir des organisations qui administrent le capital : elles ont pour tâche corollaire l'administration du travail et sont traversées des mêmes rapports hiérarchiques. Dans la mesure où le politique a pris l'ascendant sur l'économie, abolissant par-là le caractère régulateur d'institutions comme le marché, il n'y a de travail et de chômage qu'organisé. Les institutions syndicales, consciemment ou non, prennent part de l'organisation générale qui consiste à répartir et distribuer la 
plus-value. Devenues des organisations de masse, épousant alors «les vicissitudes de l'économie $»^{38}$, elles sont un relais de la domination sur le travail. Horkheimer poursuivra cette idée dans la suite de ses travaux : «De nos jours, la productivité d'un travailleur n'est pas seulement achetée par l'usine et subordonnée aux exigences de la technologie, elle est répartie et gérée par les dirigeants des syndicats ouvriers $»^{39}$. Et les protestations éventuelles, venant par exemple d'individus qui n'ont pas encore été intégrés, s'aperçoivent rapidement que « le but originel : l'abolition de la domination et de l'exploitation sous toutes ses formes, n'est encore dans la bouche des fonctionnaires qu'une phrase de propagande $\aleph^{40}$. Les inégalités, qui sont nécessaires au système hiérarchique de la bureaucratie, sont planifiées elles aussi. Les organisations ouvrières prennent part de ce mouvement du capitalisme d'État jusqu'à une spécialisation même de la fonction de stratège politique. Une couche bureaucratiquement privilégiée de spécialistes de l'organisation syndicale se consolide, au-dessus des prolétaires. Les bénéfices que ses membres en retirent ne laissent aucun doute sur leur intégration dans le capitalisme, quoiqu'ils n'aient pas besoin de prendre une part économique aux entreprises que, par la gestion de la force de travail, ils administrent :

«Le fait que les syndicats ouvriers sont organisés de manière monopoliste ne signifie pas que leurs membres - mise à part l'aristocratie ouvrière - sont des monopolistes. Cela veut dire que les leaders contrôlent la main-d'œuvre comme les directeurs des grandes sociétés anonymes contrôlent les matières premières, les machines et autres éléments de la production. Les leaders ouvriers administrent la force de travail, la manipulent, font sa publicité et essaient de fixer son prix aussi haut que possible. En même temps, leur propre pouvoir économique et social, leurs situations et leurs revenus, tous infiniment supérieurs au pouvoir, à la situation, aux revenus du travailleur individuel, dépendent du système industriel. $~^{41}$

19 Cette planification bureaucratisée générale, qui doit assurer la distribution tant de la plus-value que des inégalités et s'assurer de l'atomisation des individus, peut notamment s'appuyer sur les médias :

«Veiller à ce qu'entre les couches dominées il y ait des différences économiques, que ce soit entre ouvriers ordinaires et ouvriers qualifiés ou entre les sexes, ou entre les races, veiller à ce que l'isolement des individus soit pratiqué systématiquement à l'aide de tous les moyens de communication : journal, cinéma, radio, voilà ce qui relève du catéchisme de l'art de gouverner de façon autoritaire. »

C'est ici que s'intègre la question de l'industrie culturelle. Dans le chapitre central de $L a$ dialectique de la raison, plusieurs passages s'appuient directement sur les débats de l'Institut à propos des mutations du capitalisme depuis sa phase libérale et sur leur prolongement dans l'analyse que propose Horkheimer de l'État autoritaire. La transformation de l'art en "culture ", par l'industrie culturelle traduit le déclin de l'économique et des fonctions médiatrices du marché au profit du politique :

«L'art a imposé certaines limites au bourgeois aussi longtemps qu'il coûta de l'argent. C'en est fini désormais. Maintenant que l'art ne connaît plus de limites et que l'argent a perdu sa fonction médiatrice, il achève d'aliéner ceux qui s'en approchent et s'assimilent à lui : on aboutit à la réification totale. $»^{42}$

Plusieurs passages du chapitre Kulturindustrie, ainsi que son prolongement dans la partie qui a été finalement retirée du tapuscrit final comme de l'édition de 1947 - mais parue depuis en appendice sous le titre "Das schema der Massenkultur ${ }^{43}-$, rappellent en effet qu' « aujourd'hui, le marché libre est en train de disparaître » ${ }^{44}$, au profit d'une planification qui nécessite à la fois une grande organisation ainsi qu'une hiérarchisation et une spécialisation des tâches. 

concerne la description de la transposition au niveau de la vie quotidienne des transformations politiques et économiques contenues dans la thèse de l'État autoritaire. Et la transformation de l'œuvre d'art en industrie culturelle en est l'exemple paradigmatique. La première est en effet vue comme l'aboutissement, dans une forme symbolique, de la période bourgeoise caractérisée par le libre-échange et qui avait permis de maintenir une autonomie relative de la sphère de l'art par rapport aux autres sphères de la vie. Certes, elle portait en elle les limites que portait la structuration économique qui la caractérise : sa production comme sa réception étaient la prérogative d'individus en situation de privilège économique et social, au détriment de la souffrance de tous ceux qui avaient à endurer des tâches autrement plus pénibles pour permettre de tels privilèges. Et si, dans le meilleur des cas, les plus grands artistes «n'ont jamais été ceux qui incarnaient le style le plus pur et le plus parfait, mais ceux qui, dans leurs œuvres, utilisèrent le style pour se durcir eux-mêmes contre l'expression chaotique de la souffrance comme vérité négative ${ }^{45}$, témoignant ainsi de cette violence de la domination, la consolation n'en demeure pas moins bien maigre! Toutefois, de la même manière que le passage à l'État autoritaire découle d'une critique authentique mais trompée des inégalités induites par le capitalisme libéral, dans le cadre de l'industrie culturelle, "l'abolition d'une culture pour privilégiés [...] n'introduit pas les masses dans les sphères dont elles étaient exclues auparavant, mais entraîne justement, dans les conditions sociales actuelles, le déclin de la culture et fait progresser l'incohérence barbare dans les esprits ${ }^{46}$. En absorbant dans une même sphère cet art bourgeois, sérieux, et le divertissement de l'art populaire qui en était corollairement la "mauvaise conscience", l'industrie culturelle a annihilé la prétention à la vérité du premier et l'amusement véritable que procurait le second. En cela, l'industrie culturelle a opéré un dépassement des deux vers la réalisation d'une totalité de la culture, mais fausse ${ }^{47}$, comme l'État autoritaire est une " parodie de société sans classes ». Les mouvements artistiques critiques comme l'expressionnisme ou le surréalisme qui, par leur tentative de subversion du style, ont dénoncé cette fausseté de l'apparence de l'œuvre d'art bourgeoise comme extorquée à une domination violente laissant aux masses le seul divertissement, et n'ont reçu comme réponse que l'avènement de l'industrie culturelle, sont comparables aux authentiques syndicalistes qui, pour leur critique du capitalisme libéral, ne se sont vu qu'offrir en retour l'État autoritaire. Dans les deux cas, ils ont été intégrés, et leur existence critique n'en reste pas moins essentielle au système mais à titre de discours de "propagande »: "La dissidence réaliste devient la marque de fabrique de celui qui apporte une idée nouvelle à l'entreprise ». C'est ainsi par exemple que l'absurde, qui pouvait caractériser le geste subversif du dadaïsme, est devenu le langage vernaculaire des textes de chansons à succès ${ }^{48}$.

23 Car si, comme nous le disions en introduction de ce chapitre, la première marque civilisationnelle de la société de masse, qui dépasse de loin la sphère de la production artistique, est celle de la similarité, elle est rapidement secondée par une autre: la production contrôlée de la différenciation et de la qualité, annihilant par là même la possibilité de ces dernières. C'est le sens du célèbre passage : « [i]l a été prévu quelque chose pour chacun afin que nul ne puisse échapper, les différences sont mises en relief et diffusées partout $\aleph^{49}$. Même intégrée, la culture doit rester un élément essentiel par lequel la subjectivité individuelle trouve à s'exprimer. Ou plutôt à exister car le but n'est pas tant de laisser s'exprimer telle subjectivité que de la produire et de la 
contraindre à se former sur le modèle particulier qui lui est destiné, lui-même construit sur le modèle général ${ }^{50}$. À l'époque de l'État autoritaire, la culture s'assure ainsi que chacun soit à sa place, en lui offrant le service de "tout schématiser pour lui ${ }^{51}$, avec pour corollaire de schématiser également sa position dans la hiérarchie sociale. L'industrie culturelle peut ainsi créer des différences de qualité mais qui ne sont qu'un voile du fait que toutes les gammes de qualités sont de simples versions du même modèle. On peut en trouver un exemple éclairant, dans les Minima Moralia, pour ce qui concerne la production automobile :

«Alors que la Cadillac a sans aucun doute autant d'avantages de plus sur la Chevrolet que son prix est plus élevé, cette supériorité [...] résulte elle-même d'un plan d'ensemble qui équipe habilement la première de meilleurs cylindres, la seconde de cylindres, de vis et d'accessoires plus médiocres, sans que le schéma de base du produit de masse s'en trouve modifié : il suffirait de quelques décalages dans la production pour transformer une Chevrolet en Cadillac. $»^{52}$

Ne dissimulant pas son intégration à la sphère économique, elle-même administrée bureaucratiquement par le politique, l'industrie culturelle ne cesse de recréer des illusions de libre concurrence et de libre choix. Mais il ne faut pas s'y tromper, « [l]e fait d'offrir au public une hiérarchie de qualités n'a pour but qu'une quantification d'autant plus parfaite $»^{53}$. Voilà un trait essentiel du passage du libéralisme à l'État autoritaire (qu'il soit démocratique ou totalitaire), résumé en une métaphore que l'on trouve également dans les Notes critiques de Horkheimer, celle du passage du téléphone à la radio : « libéral, le téléphone permettait encore à l'abonné de jouer le rôle d'un sujet. Démocratique, la radio transforme tous les participants en auditeurs et les soumet autoritairement aux programmes des différentes stations qui se ressemblent tous ${ }^{54}$. La radio porte ainsi la dimension autoritaire qu'on retrouve aussi bien dans la société démocratique des États-Unis que dans l'Allemagne nazie: «Aux États-Unis, elle ne perçoit aucune redevance du public et prend ainsi l'aspect trompeur d'une autorité désintéressée et imparfaite comme faite sur mesure pour le fascisme $»^{55}$. Malgré la " pseudo-individualisation ${ }^{56}$, la standardisation qu'impose la planification dans l'État autoritaire tend à viser la production de conduites psychiques elles-mêmes standardisées, risquant en permanence de tomber dans le totalitarisme: «Dans l'industrie culturelle, l'individu n'est pas seulement une illusion à cause de la standardisation des moyens de production. Il n'est toléré que dans la mesure où son identité totale avec le général ne fait aucun doute ${ }^{57}$.

Comme on le comprend également dans ces extraits, il est, à propos de la notion d'industrie culturelle telle qu'elle est conceptualisée dans La dialectique de la raison, une importante dimension de critique de la technique en tant que forme nouvelle de la rationalité. «Rationalité de la domination même » qui constitue « le caractère coercitif de la société aliénée ${ }^{58}$, la rationalité technique conditionne la forme des productions de l'industrie culturelle qui, en la véhiculant, étendent en retour son autorité sur l'ensemble du monde social. Mais il ne s'agit pas tout à fait d'une critique autonome de la technologie en tant que telle. Bien plutôt, le concept d'industrie culturelle, avec son syntagme en forme d'oxymore, est surtout une critique de l'emploi de la technique, qui ne se résume aucunement au progrès proprement technologique. Dans ce cadre, à nouveau, il vise bien au-delà de la sphère proprement culturelle pour interroger le principe même du fordisme ou de la production de masse dans sa constitution de la planification et, partant, de la nouvelle forme de capitalisme menant à l'État autoritaire. Ainsi, et toujours à la suite du texte sur ce dernier, les auteurs dénoncent la 
perversion historique de l'aspiration légitime à une répartition plus égalitaire de la production entre les individus que matérialise l'ordre technique. La réponse à cette aspiration n'a pas été une meilleure répartition mais une intensification de la production, doublée des mesures de charité de l'État providence ${ }^{59}$. La technique est alors devenue l'excuse d'un progressisme charlatan: la nécessité de satisfaire le maximum de personnes «impose des méthodes de reproduction qui, à leur tour, fournissent en tous lieux des biens standardisés $»^{60}$. Il faut donc bien des modèles et une structuration du management, afin de choisir les meilleurs standards et d'en planifier production et distribution en utilisant au mieux les possibilités de la technique : voilà l'égalitarisme vulgaire que l'industrie culturelle vise à atteindre au moyen de son régime technique. "Mais ce que l'on ne dit pas, c'est que le terrain sur lequel la technique acquiert son pouvoir sur la société est le pouvoir de ceux qui la dominent économiquement. $»^{61} \mathrm{Et}$ c'est bien, fondamentalement, ce pouvoir-là qui est visé et qui se dissimule derrière sa propre excuse concernant la nécessité d'employer la rationalité technique : celui de la "concentration économique et administrative». La technique sert ainsi au « capital qui se tient derrière elle $»^{62}$ à la fois de modèle de rationalité pour la production industrielle et de propagande culturelle, qui est néanmoins percée à jour lorsqu'on considère le caractère irrationnel des tâches auxquelles elle est employée :

«L'idée de la pleine exploitation des possibilités techniques existantes, de la pleine utilisation des capacités en vue de la consommation massive de biens esthétiques, fait partie d'un système économique qui refuse pourtant d'utiliser les ressources disponibles lorsqu'il s'agit de faire disparaître la faim dans le monde. » $^{63}$

On constate ainsi que, dans la version qui en est proposée dans le chapitre central de $L a$ dialectique de la raison, le concept d'industrie culturelle vise à rendre compte des transformations culturelles consécutives aux mutations du capitalisme qui ont été étudiées par l'Institut für Sozialforschung au début des années 1940 et notamment telles qu'elles ont été condensées dans l'étude sur l'« État autoritaire ». L'élément majeur de l'analyse en est la fin de la libre concurrence et le passage à l'ère des monopoles. Mais si la catégorie de "monopole » elle-même a peut-être été quelque peu gommée de ce chapitre pour la publication définitive, laissant entendre une distanciation définitive d'avec la conception de Neumann, il n'en est pas moins difficile de considérer pour autant que dans sa version de 1947, le concept d'industrie culturelle réfléchit en termes de primauté du politique. Comme dans le cadre des travaux sur l'État autoritaire, la position $\mathrm{du}$ texte intègre évidemment l'importance nouvelle du politique, chère à Pollock, mais en appuyant toutefois - quoique de manière moins systématisée -, un ensemble de remarques concernant une primauté de l'économique qui reste le fil rouge de l'analyse. La caractéristique socioéconomique primordiale qui est pointée en est le processus de bureaucratisation, qui constitue l'intégration des deux.

La fin du chapitre montre de manière parlante la finesse des rapports entre économie et politique à l'heure de l'État autoritaire à partir de l'analyse de la publicité, "qui devient d'autant plus omnipotente qu'elle paraît absurde sous un monopole ${ }^{64}$. En effet, la publicité s'expliquait, à l'époque du capitalisme libéral, par le besoin d'« orienter le consommateur sur le marché ». Permettant au "meilleur* fournisseur, fût-il inconnu, de trouver le bon consommateur pour sa marchandise $»^{65}$, elle était finalement garante de la concurrence, dont elle constituait un service ad hoc, afin de faire gagner du temps à l'acheteur. Mais à l'époque des monopoles, elle devient un pur outil au service du resserrement de la domination qu'exercent les trusts sur leurs clients. Alors, 
«Seul celui qui peut payer les droits exorbitants que réclament les agences de publicité [...], c'est-à-dire ceux qui font déjà partie du système ou sont cooptés par les décisions du capitalisme bancaire ou industriel, peuvent pénétrer comme vendeurs sur ce qui n'est plus qu'un pseudo-marché. Les frais de publicité qui refluent finalement dans les poches des trusts, évitent d'avoir à éliminer des outsiders indésirables dans une concurrence acharné; pour les patrons ces frais sont la garantie qu'ils resteront entre eux ; à peu près comme dans les délibérations des conseils économiques qui, dans les États totalitaires, contrôlent l'ouverture de nouvelles entreprises et la gestion de celles existant déjà. De nos jours, la publicité est un principe négatif, un dispositif de blocage; tout ce qui ne porte pas son empreinte est économiquement suspect. $»^{66}$

sien comprendre cet extrait où s'enchevêtrent les différentes dimensions politiques et économiques. Dans les mutations du capitalisme post-libéral, la publicité a changé de fonction : elle n'a plus un rôle essentiellement économique et elle dissimule à peine un "pseudo-marché ", selon la thèse pollockienne du capitalisme d'État. Mais cette nouvelle fonction, qui s'apparente à un outil de contrôle politique, est au service d'une domination économique où le pouvoir est exercé par les patrons des plus importants monopoles, ou des institutions bancaires qui les choisissent. On voit donc que, en dernière instance, comme dirait Engels, « les motifs sont, au fond, économiques » 67.

Pour Horkheimer et Adorno, la publicité est devenue le paradigme de l'industrie culturelle, son "élixir de vie ", ce qui révèle combien le fonctionnement de la première, entre économie et politique, est au cœur du mécanisme de la seconde. C'est donc bien, «le triomphe du capital investi $»^{68}$ qui est le «contenu réel » de tous les produits de l'industrie culturelle ainsi que le cœur de ce que signifie leur standardisation, et cela, quels que soient «l'intrigue choisie par la direction de la production», pour le cas des produits culturels les plus narratifs, ou le discours de propagande qui les accompagne, pour les autres. L'industrie culturelle décrit cette culture qui, dans les mutations du capitalisme et pour des raisons économiques qui restent déterminantes, accompagne la prise d'ascendant du politique dans le contrôle de la vie sociale, à tel point que, comme le rappelle Adorno dans le "retour » sur le concept qu'il a proposé au début des années 1960 , «se mue en public relations à savoir la fabrication d'un good will tout court, sans égard à des producteurs ou des objets de vente particuliers ${ }^{69}$.

\section{L'industrie culturelle comme catégorie d'une critique de l'économie politique adornienne}

De ce point de vue, l'industrie culturelle semble contenir, dès le chapitre écrit à quatre mains, un élément fondamental qui dépasse les analyses sur l'État autoritaire (ou la question du capitalisme d'État) et annonce des réflexions économiques ultérieures d'Adorno. Il est ainsi intéressant de l'étudier en rapport avec la continuité des travaux du philosophe, afin de voir à la fois combien ces derniers influent sur les différentes reprises du concept, mais aussi combien l'industrie culturelle oriente d'autres de ses réflexions sociologiques et économiques. Toujours dans son « retour » des années 1960 sur le concept, Adorno rappelle que «[1]e consommateur n'est pas roi, comme l'industrie culturelle le voudrait, il n'est pas le sujet de celle-ci, mais son objet $»^{70}$. Poussons plus avant cette étude du sujet. Qui seraient, finalement, les sujets de l'industrie culturelle? Comme on vient de le voir, dans le chapitre écrit avec 
Horkheimer, s'il est fait référence à des dominants (les " autorités exécutives " et " directeurs généraux $»^{71}$, les « managers de l'économie » ${ }^{72}$, etc.), il ne s'agit que d'une élite bureaucratique qui n'en est pas moins dominée elle-même par le " capital investi » qui règne seul en maître sur l'industrie culturelle. C'est lui qui choisit la place accordée à chacun, du garçon-coiffeur jusqu'au Führer, sur sa capacité à s'identifier complètement au modèle général. Mais qui se cache derrière ce «capital investi »? Lorsqu'il est question d' intérêts privés ${ }^{73}$, ceux-ci ne font partie que d'une mécanique impersonnelle qui est elle-même un rouage du système. L'appendice au chapitre Kulturindustrie cite à cet égard le Zarathoustra de Nietzsche - citation qu'Adorno reprendra dans un de ses derniers textes sur le capitalisme tardif (cf. ci-après) - : « Pas de berger et un seul troupeau $\aleph^{74}$. La logique anonyme du capital, qui administre l'industrie culturelle, reste invisible et se cache derrière des administrateurs. Ceux-ci forment une élite anonyme, mais n'en sont pas moins également dominés par la même logique. En cela, la similarité qui, comme nous le rappelions en introduction, est la marque essentielle de la civilisation de masse, finit par toucher jusqu'à tous les individus de la société ainsi administrée. C'est là qu'elle produit son effet le plus pervers: la perte de la catégorie même d'individu dans le mouvement même de reproduction de la société :

" Aujourd'hui, l'industrie culturelle a pris en charge la fonction civilisatrice de la démocratie des asservis et des chefs d'entreprise, qui n'avait pas non plus un sens très affiné des déviations intellectuelles. Tous sont libres de danser et de s'amuser tout comme, depuis la neutralisation historique de la religion, ils sont tous libres d'entrer dans une des innombrables sectes existant. Mais la liberté dans le choix de l'idéologie, qui reflète toujours la coercition économique, apparaît dans tous les secteurs comme la liberté de choisir ce qui est toujours semblable. $»^{75}$

Le caractère abstrait de la domination qui se traduit dans l'industrie culturelle est un thème important des travaux d'Adorno. Dès 1942, le philosophe proposait au sein de l'Institut de Francfort un article de "[r]éflexions sur la théorie des classes", dans lequel il critiquait la croyance en la constitution inévitable du socialisme par le prolétariat en tant que classe historique consciente d'elle-même. À la suite de ses collègues Pollock et Horkheimer à la même époque, Adorno attaque notamment l'importance de la théorie de la paupérisation pour la doctrine marxiste des classes. Alors que le capitalisme est sorti de sa phase libérale, et que l'importance progressive de la dimension politique y a réduit le temps de travail et instauré des protections contre le chômage et l'extrême précarité, la pauvreté ne peut plus être considérée comme un levier déclencheur de l'action révolutionnaire. On peut voir toutefois, dès ce texte rédigé en pleine discussion sur le capitalisme d'État, combien Adorno essaie déjà de faire la synthèse entre les conceptions de Pollock et un maintien des catégories marxistes plus classiques ${ }^{76}$ : sous le règne des monopoles, "[1]a classe dominante disparaît derrière la concentration du capital. Cette dernière a atteint une ampleur, un poids net, qui permet au capital de se présenter comme une institution, comme l'expression de la société dans son ensemble». En cela «se parachève l'antique caractère fétiche de la marchandise, qui réfléchit des relations entre hommes sous la forme de relations entre choses $»^{77}$.

Mais il subsiste néanmoins, dans ce texte de 1942, une certaine indétermination sur la spécificité de l'économie capitaliste, visible dans cette étrange expression de caractère fétiche "antique ». La "domination la plus récente » est vue comme la survivance d'une « antique domination » - i.e. la guerre entre clans rivaux - « qui s'est infiltrée 
dans l'appareillage $\aleph^{78}$. Le capitalisme est considéré comme une cristallisation spécifique de l'histoire de la domination: la bourgeoisie, qui a pris le pouvoir avec l'aide massive du reste du tiers-état, a établi son pouvoir sur l'exploitation d'une marchandise spécifique: la force de travail. Elle a habillé cette domination d'un discours d'égalité trompeur afin justement de viser l'universalisme pour convaincre les couches inférieures du féodalisme de s'y rallier. Mais les succès des «libres concurrents» dépendent «du pouvoir politique et social qu'ils représentent, des pillages anciens et nouveaux de l'exploitation coloniale, d'une accointance avec la propriété féodale - que l'économie concurrentielle n'a jamais sérieusement liquidée -, ainsi que du rapport à l'appareil de domination directe qu'est l'armée $»^{79}$. En cela on peut dire que «[1]'économie [comprise comme science bourgeoise ayant visé une théorisation de la domination sur le capital en vue de sa reproduction, CM] est un cas particulier de l'économie, entendue au sens du manque organisé en vue de la domination $»^{80}$. Adorno pose ici l'importance des modes de production précapitalistes dans la préparation de la domination, cette dernière étant simplement perpétuée, par d'autres moyens, dans le capitalisme. Les monopoles sont considérés comme entités extra-économiques qui poursuivent l'histoire de la domination clanique et, partant, comme parachèvement du fétichisme en tant que catégorie d'ordre transhistorique.

Adorno n'y pressent pas moins cette particularité de la domination dans le capitalisme des monopoles : son caractère abstrait, qui ne découle pour autant pas d'une ignorance ou d'un manque de connaissance de la part des individus (comme c'était le cas dans les modes de production précédents). Cette particularité est rapprochée de la division du travail qui y est " poussée à l'extrême », jusqu'à ce que " du fait de leur déqualification, elle a en même temps de plus en plus rapproché les unes des autres les tâches singulières, à tel point que celui qui en connaît une les connaît virtuellement toutes $»^{81}$ mais sans aller jusqu'à abolir le travail. Le processus de travail façonne donc les individus et leur intégration consiste à devenir identiques aux autres, et ce sans besoin de les « influencer " par des croyances chimériques ou idées fausses. Adorno pose ainsi déjà la «culture de masse $»^{82}$ que peut représenter la radio et des films comme conséquence civilisationnelle qui contribue à faire des hommes «simplement, encore et toujours, ce qu'ils sont de toute façon déjà sous la contrainte du système ${ }^{83}$.

Le raffinement de l'analyse de ce caractère abstrait de la domination, qui se double d'un égalitarisme fallacieux, sera un objet essentiel des travaux de sociologie d'Adorno, à partir de l'après-guerre, et de la critique de l'économie politique qui les essaime de manière éparse - n'ayant pas fait chez le philosophe l'objet d'une systématisation dans un ouvrage spécifique ${ }^{84}$. Exploration de l'intérieur de la vie quotidienne américaine, les Minima Moralia, composés en parallèle de la rédaction de La dialectique de la raison, et publiés en 1951, apportent ainsi une précision importante: "dans la société de l'échange*, le sujet n'en est pas un [...] il n'est qu'objet de cette société $»^{85}$. Adorno revient au fétichisme de la marchandise pour caractériser la société à l'heure de l'industrie culturelle. Son principe est celui de l'échange, qui rend toutes les choses équivalentes, à commencer par la force de travail des individus elle-même, marchandise-clef d'une telle société :

" C'est seulement lorsque le processus qui s'engage avec la transformation de la force de travail en marchandise et qui pénètre les hommes de toutes parts objectivant chacune de leurs impulsions pour les rendre commensurables comme une forme entre autres des relations d'échange - que sont réunies les conditions permettant à la vie de se reproduire dans les rapports actuels de la production. $»^{86}$ 
La «relation d'échange " (Tauschverhältnis), catégorie centrale de la réflexion socioéconomique d'Adorno, en vient donc à caractériser plus spécifiquement la société capitaliste. Sous le règne de la marchandise, toutes les activités sont considérées comme purs moyens et réduites "à un temps de travail abstrait et interchangeable ", à tel point que « [l]a qualité des choses cesse d'être leur essence et devient l'apparition accidentelle de leur valeur $\aleph^{87}$. Bon nombre de passages reprennent ainsi ou paraphrasent Marx pour appuyer le devenir abstrait de toute qualité dans la société de l'échange, qui détermine la direction que prend ses évolutions, jusqu'à l'ère des monopoles où «[i]l ne vient à l'idée de personne qu'il pourrait exister des prestations intraduisibles en valeur d'échange $»^{88}$.

Le concept d'industrie culturelle devient alors petit à petit une pièce maitresse de la critique de l'économie politique adornienne. Et celle-ci s'appuie de manière centrale sur la critique marxienne de l'économie politique qui signifie, comme Adorno l'annonce dans un séminaire estival de 1962 consacré à Marx, à la fois critique de la théorie classique du libéralisme et critique de l'économie elle-même et de ses catégories ${ }^{89}$. Traducteur en français de cette conférence sur Marx de l'été 1962, David Buxton propose, dans son commentaire, de la mettre en rapport avec le «retour» sur le concept d'industrie culturelle énoncé lors de diverses émissions radiophoniques la même année, bien que ces allocutions semblent probablement être le texte le moins économique du philosophe sur la question. Ce rapprochement nous apparaît judicieux.

En effet, dans la première, Adorno rappelle, en revenant sur les catégories fondamentales de la critique marxienne, les principes de fonctionnement de la société capitaliste qui est caractérisée par l'échange. On sait que, d'après Marx, la marchandise est la "forme cellulaire» de la société capitaliste qui se présente comme une accumulation de marchandises. La particularité essentielle de ces dernières, qui les différencie de l'ensemble des biens produits dans d'autres sociétés humaines, est qu'en plus de leur valeur d'usage, par laquelle elles satisfont des besoins, elles possèdent, justement, une valeur d'échange, qui «n'est pas dérivée du besoin » et par laquelle elles peuvent être échangées contre d'autres objets. "Ce qui rend la marchandise échangeable, c'est l'unité de temps de travail social abstrait ${{ }^{90}}^{90}$, seule chose que deux marchandises différentes et par nature incommensurables ont en commun. Ainsi la « valeur » des marchandises, qui permet d'en calculer entre elles les valeurs d'échange, dépend pour chacune du temps de travail moyen qui, eu égard au niveau de développement des forces productives dans une société donnée, est nécessaire pour les produire. Mais dans le capitalisme, le travail est lui-même une marchandise : celle qu'une classe particulière, les travailleurs, proposent « librement » à la vente. En tant que marchandise, le travail possède également une valeur d'usage et une valeur d'échange et la différence entre les deux est la source de la survaleur. Car, lorsqu'un capitaliste achète la marchandise-force de travail d'un prolétaire contre un salaire (sa valeur d'échange), il peut en faire usage comme bon lui semble et la faire travailler plus que le temps nécessaire à la stricte reproduction de ce qu'il a dépensé pour l'acquérir. "C'est là que se trouve la source de la survaleur, sans que l'on doive prendre en considération la marchandise produite. [...] Derrière cela se trouve la totalité des relations de classe. Le travailleur accepte ces conditions seulement parce qu'il n'a rien d'autre que sa force de travail. $»^{91}$ cette société, «ce qui est décisif, c'est la dominance de l'appareil de production par 
rapport aux besoins » qui ne sont satisfaits que collatéralement. Toutes les relations y prennent en conséquence la forme de l'échange de marchandises. "Ce qui est une relation sociale figée au sein de la marchandise est perçu comme s'il s'agissait d'une qualité naturelle, d'une existence de choses en elles-mêmes $\|^{92}$. La relation prend la forme d'un échange d'équivalents: on réduit ce qui est incommensurable à de l'identique. D'un autre côté, afin d'augmenter l'accumulation de survaleur, les capitalistes sont "poussés à développer des machines afin de remplacer le travail vivant par le travail mort $»^{93}$. Ils y ont intérêt car les machines (travail mort) permettent des gains de productivité au niveau de chaque force de travail employée (soit une augmentation de la valeur d'usage de celle-ci). Ainsi, chaque innovation technique introduite par un capitaliste lui apporte un surplus momentané de survaleur: le temps de travail nécessaire pour produire ses marchandises passe momentanément sous le temps moyen habituellement employé à produire des marchandises de même type qui sert d'étalon de calcul de leur valeur. Mais ce gain est de courte durée : rapidement, tous les concurrents sur le même marché se trouvent obligés d'introduire une innovation technique comparable. "S'ils ne le font pas, ils périront dans la lutte concurrentielle $»^{94}$. Si bien qu'en peu de temps, c'est le temps de travail moyen de production de la marchandise qui diminue, c'est-à-dire, finalement, la valeur de la marchandise elle-même dont le temps de travail moyen est l'unité de calcul: il faut désormais produire une plus grande quantité de marchandise pour réaliser la même valeur. Les capitalistes sont toujours incités à développer d'autres machines à tel point qu'une part toujours grandissante de capital est investie dans ces dernières et une part toujours plus faible consacrée à employer du travail vivant. Mais c'est le travail vivant qui est la seule source de survaleur : «les machines ne peuvent produire de la valeur. Ce que les machines produisent renvoie au travail car elles sont elles-mêmes produites par le travail». Il faut toujours un travailleur pour exploiter le potentiel productif de la machine. En conséquence, le capital a toujours intérêt à étendre son emprise à de nouveaux secteurs et les asservir petit à petit à la loi de la marchandise, c'est-à-dire à y orienter la production selon son procès d'abstraction de l'activité productrice, de sa transformation en travail et de son exploitation. Et même ce faisant, à cause du remplacement progressif du travail vivant par du travail mort, «de plus en plus, une quantité de travail devient surnuméraire, créant ainsi des conditions de crise et d'une menace sans cesse grandissante au système lui-même ${ }^{95}$.

Or, dans son retour de la même année sur l'industrie culturelle, Adorno rappelle combien il faut «faire dériver le phénomène de l'économie, de la recherche de nouvelles possibilités de faire fructifier le capital dans les pays hautement industrialisés. Les anciennes possibilités deviennent de plus en plus précaires du fait de ce même processus de concentration qui de son côté rend seul possible l'industrie culturelle en tant qu'institution puissante $»^{96}$. Quelle est la particularité de la période " actuelle "? D'un côté, comme on l'a vu, elle exige de nouveaux secteurs où étendre la loi de l'échange et de la marchandise. La culture a été, tout au long du vingtième siècle, le domaine privilégié de cette extension. Jusqu'à ce que, petit à petit, les processus spécifiques de la production y soient transformés et que «[1]a praxis entière de l'industrie culturelle applique carrément la motivation du profit aux produits

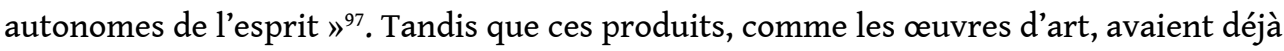
été conquis par la marchandise, ils avaient néanmoins réussi à limiter cette conquête à la seule sphère de leur distribution. À l'époque de l'industrie culturelle «les productions de l'esprit [...] ne sont plus aussi des marchandises mais le sont 
intégralement». C'est notamment dans l'emploi de la technique que la différence se révèle. Tandis que, dans les œuvres d'art, la technique "se rapporte à l'organisation immanente de la chose, à sa logique interne ${ }^{98}$ - bref, qu'elle est utilisée en rapport dialectique avec la forme historique des œuvres - dans l'industrie culturelle, elle est appliquée de l'extérieur, selon les besoins d'innovation techniques que la production de valeur exige dans la société de l'échange et concerne principalement la distribution et la reproduction mécanique. En même temps, l'industrie culturelle est, via l'attitude qu'elle engendre (et qui ne se réduit pas à ses «messages " ${ }^{99}$ ), ce par quoi l'ordre de l'extension de la logique de la marchandise est transposé dans les subjectivités des individus. Parce que ses productions sont toujours consommées comme autrefois étaient consommées les œuvres d'art, l'industrie culturelle induit cette attitude de réception cultuelle (qui caractérisait historiquement la réception des œuvres de la tradition, comme l'explique Walter Benjamin dans son célèbre essai ${ }^{100}$ auquel Adorno fait référence) pour un contenu qui est de part en part marqué par l'ordre de la marchandise. Ainsi, dans une autre conférence donnée en 1965, Adorno explique que

«L'industrie culturelle est née de la tendance qu'a le capital à tout mettre à profit. Elle s'est développée sous le règne de la loi du marché, sous la contrainte de l'adaptation à ses consommateurs; mais elle s'est renversée ensuite pour devenir l'instance qui fixe et renforce la conscience dans chacune de ses formes en vigueur, qui constituent le statu quo intellectuel. ${ }^{101}$

On voit combien, finalement, l'industrie culturelle est la pierre angulaire du capitalisme " post-libéral ». Cela n'empêche que celui-ci consiste toujours en les mêmes rapports de domination que ceux que Marx analyse dans sa critique de l'économie politique. En cela, l'un des derniers textes d'Adorno, celui de la conférence inaugurale prononcée en 1969 à la $16^{\mathrm{e}}$ Journée des sociologues allemands réalise une synthèse exceptionnelle. Le philosophe y pose la question de savoir si l'analyse de Marx est dépassée, comme le prétendent bon nombre de sociologues de l'époque en affirmant que «le développement industriel a [...] rendu caducs le concept de capitalisme lui-même, la différence entre États capitalistes et non capitalistes, voire la critique du capitalisme » ${ }^{102}$. La réponse d'Adorno est claire. Certes, la technique domine la production de part en part, à tel point que le travail industriel est « le patron commun de la société $»^{103}$. Les processus de travail spécifiques de l'industrie se sont propagés «suivant une nécessité économique dans les domaines de la production matérielle, de l'administration, dans la sphère de la distribution et dans celle qui se nomme culture ${ }^{104}$. Certes, la grande profusion de biens de consommation qui en a résulté a contribué à limiter largement les effets de la paupérisation des classes inférieures dans les pays occidentaux où « les différences de classe ressortent, en termes de niveau de vie et de conscience, de manière beaucoup moins visible que cela n'a été le cas pendant les décennies de la révolution industrielle et celles qui ont suivi $»^{105}$. Les membres des classes inférieures ont également en grande partie intégré la vision du monde de la société bourgeoise à tel point que des théoriciens subjectifs se demandent si l'on peut encore parler de classe - à mesure que la conscience de classe décroît. Mais la théorie ne peut se limiter à une telle présentation des faits « qui, de leur côté, contribuent certes beaucoup à la critique mais qui, selon la théorie critique, dissimulent tout autant la structure $»^{106}$. D'une part, certaines enquêtes, qui ne se limitent pas à enregistrer des faits par l'application de méthodologies prescrites démentent toujours ces faits. D'autre part, un mode de production se définit par la combinaison des forces productives, d'un côté (i.e. la force de travail déployée par les travailleurs, selon des modes d'organisation 
spécifiques du travail, ainsi que les matières premières et instruments de production) et des rapports de production (i.e. l'organisation sociale autour de la production). Et si, dans la théorie marxiste, les contradictions entre les deux conditionnent le saut qualitatif d'un mode de production à un autre, car les rapports de production doivent être en phase avec le niveau de développement des forces productives, il ne faudrait pas trop vite présumer qu'une modification d'un de ces deux facteurs implique le dépassement du système. Aussi, si « la société actuelle est sans aucun doute une société industrielle si l'on s'en réfère au niveau de ses forces productives ", il n'en demeure pas moins qu'elle « relève en revanche du capitalisme quant à ses rapports de production $»^{107}$. Ainsi, «[1]es hommes sont toujours ce que, d'après l'analyse marxienne, ils étaient vers le milieu du XIXe siècle: des appendices de la machinerie ${ }^{108}$. Mais il y a une particularité supplémentaire : désormais, la nécessité de faire du profit s'est objectivée, « les besoins sont devenus pour de bon - ils l'étaient depuis longtemps en puissance des fonctions de l'appareil de production et non l'inverse ». Cette objectivation rend le profit le seul véritable sujet du capitalisme :

«Depuis longtemps, les objets [du processus économique] ne sont plus seulement les masses, mais aussi ceux qui disposent des moyens de production et leurs associés. Conformément à la théorie classique, ils sont dans une large mesure devenus des fonctions de leur propre appareil de production. La question très débattue qui porte sur la managerial revolution et sur la prétendue passation de pouvoirs entre les propriétaires juridiques et la bureaucratie est, face à cela, d'une importance secondaire. $»^{109}$

41 Adorno semble ainsi répondre a posteriori aux débats qui ont animé l'Institut autour de la thèse du capitalisme d'État. Pour lui, le capitalisme continue d'opérer conformément à son concept, c'est-à-dire selon les mécanismes analysés par Marx, et ce malgré les spécificités du développement industriel. Le philosophe francfortois choisit donc de parler de «capitalisme tardif» pour caractériser cette période, qui est aussi caractérisée par l'industrie culturelle. Si elle n'a pas résulté en un accroissement de la pauvreté, comme la théorie de la paupérisation le prévoyait, elle n'en a pas moins vu s'accroitre la dépendance des individus "par rapport à un appareillage qui s'est affranchi de la conscience de ceux qui le manient $»^{110}$ et, par là même, s'étendre la nonliberté.

En restant avec les catégories marxiennes, on pourrait synthétiser de la manière suivante : le capitalisme à son stade libéral restait principalement, pour une très grande majorité d'activités, et notamment celles de l'esprit, dans le cadre de ce que Marx nomme la "subsomption formelle du travail sous le capital ", soit l'appropriation par le capital d'un procès de travail qui lui préexistait mais sans que cela n'entraîne « une transformation essentielle affectant le mode réel du procès de travail, le procès de production effectif ${ }^{111}$. La domination des capitalistes y était violente mais le travail lui-même conservait une part importante du sens précapitaliste qu'il pouvait avoir. En revanche, la recherche de profit par l'absorption de survaleur, relation fondamentale de la société de l'échange, s'est autonomisé et a conduit à un dépassement de ce stade. Le capitalisme des monopoles résulte en une civilisation industrielle dont il a par ailleurs besoin qu'elle pénètre toutes les sphères sociales et subjectives qui se soumettent peu à peu à la domination abstraite et bureaucratique de l'économie. Ce capitalisme, qu'Adorno nomme "tardif», représente quant à lui le summum de l'étape que Marx nomme «subsomption réelle du travail sous le capital » qui «transforme la nature réelle $d u$ procès de travail ainsi que ses conditions réelles »: ce n'est plus 
uniquement une domination $d u$ travail mais une domination dans le travail, et une domination par le travail qui s'étend à toutes les sphères de la vie, selon son principe capitaliste, à savoir la destruction des qualités en réduisant toute tâche à du travail abstrait. L'industrie culturelle est à la fois le lieu privilégié par lequel Adorno analyse cette extension du domaine de la marchandise (celui des œuvres de l'esprit et notamment des œuvres d'art) mais aussi une pièce fondamentale de ce système qui nécessite la reproduction des catégories de la marchandise au niveau même des subjectivités.

L'industrie culturelle ne peut donc être considérée comme la catégorie par laquelle Adorno entérine définitivement l'ascension de la dimension politique dans les sociétés du capitalisme "post-libéral » à la suite de la thèse proposée par Pollock dans les années 1940. Elle est au contraire un concept complexe, au cœur d'une pensée qui, sans négliger la dimension politique, maintient l'importance de la critique de l'économie pour analyser la société du capitalisme tardif. Si, dans le chapitre central de La dialectique de la raison écrit avec Horkheimer, elle reste une catégorie permettant de décrire la civilisation de l'État autoritaire, la suite des travaux d'Adorno la voit s'éloigner de cette simple description et révéler sa teneur propre de concept critique, notamment par un retour progressif aux textes de Marx. La similarité ou la ressemblance, qui caractérisent la civilisation de masse sont donc à considérer bien audelà de la métaphore et même de la définition technique. Certes, les automobiles Ford ressemblent à s'y méprendre aux automobiles Chevrolet. Certes, ces dernières ressemblent - à quelque maquillage « luxe " près - à des Cadillac, avec lesquelles elles partagent aujourd'hui encore, comme à l'époque où écrit Adorno, une partie de leurs standards de production au sein de la même société-mère, General Motors. Certes, le jazz de Guy Lombardo ressemble à celui de n'importe quel autre crooner de l'époque comme aujourd'hui les chanteuses pop à succès ont les mêmes voix, chantent de la même manière des tubes produits par les mêmes producteurs avec, selon la mode de l'année, les mêmes gimmicks et les mêmes sonorités. Mais la similarité concerne avant tout le caractère d'équivalence de leur valeur, ce secret des relations sociales dans le capitalisme par lequel n'importe quelle marchandise est rendue comparable à une autre qui lui est incommensurable en termes de qualité, de matérialité ou d'expérience, par la suite de la réduction du travail au travail abstrait simple mesure de la valeur des marchandises.

L'industrie culturelle en tant que concept vise à critiquer la civilisation qui correspond à ce moment historique, à partir des productions symboliques qu'elle offre, certes, car ces productions symboliques ont toujours été le témoin privilégié par lequel on analyse l'état d'une société, mais aussi et surtout à partir des expériences les plus anecdotiques de la vie quotidienne dans la société ainsi administrée par le capitalisme tardif. Ainsi, le concept d'industrie culturelle, dans la suite des travaux d'Adorno depuis La dialectique de la raison, ne sera pas uniquement un moyen de décrire l'état de la production des biens artistiques de l'après-guerre, ni uniquement une critique de la civilisation industrielle - ce qu'elle était majoritairement dans le chapitre écrit avec Horkheimer. Elle devient également une catégorie de la critique adornienne. 


\section{BIBLIOGRAPHIE}

Adorno, Theodor W. «Capitalisme tardif ou société industrielle ? » In Société: IntégrationDésintégration. Écrits sociologiques, 85-107. Paris : Payot, 2011.

Adorno, Theodor W. «L'industrie culturelle ». Communications 3, nº 1 (1964) : 12-18.

Adorno, Theodor W. Minima Moralia. Réflexions sur la vie mutilée. Paris : Payot, 2003.

Adorno, Theodor W. « Réflexions sur la théorie des classes ». In Société : Intégration-Désintégration. Écrits sociologiques, 57-84. Paris : Payot, 2011.

Adorno, Theodor W. « Société I ». In Société: Intégration-Désintégration. Écrits sociologiques, 24-36. Paris : Payot, 2011.

Adorno, Theodor W. The Culture Industry. Selected Essays on Mass Culture. New York, NY : Routledge, 1991.

Adorno, Theodor W. « Theodor W Adorno über Marx und die Grundbegriffe der soziologischen Theorie ». In Dialektik der Wertform, par Hans-Georg Backhaus, 501-13. Fribourg : Ca ira, 1997.

Benjamin, Walter. «L'đEuvre d'art à l'époque de sa reproductibilité technique (dernière version de 1939)». In CEuvres III, 269-316. Paris : Gallimard, 2000.

Braunstein, Dick. Adornos Kritik der politischen Ökonomie. Bielefeld : Transcript Verlag, 2011.

Brick, Barbara, et Moishe Postone. « Kririscher Pessimismus und die Grenzen des traditionellen Marxismus ». In Sozialforschung als Kritik, édité par Wolfgang Bonß et Axel Honneth, 179-97. Frankfurt am Main : Suhrkamp, 1982.

Genel, Katia. Autorité et émancipation. Horkheimer et la Théorie critique. Paris : Payot, 2013.

Horkheimer, Max. Éclipse de la raison. Paris : Payot, 1974.

Horkheimer, Max. « L'État autoritaire ». In Théorie critique, 301-25. Paris : Payot, 1978.

Horkheimer, Max, et Theodor W. Adorno. « Das Schema der Massenkultur ». In Dialektik der Aufklärung. Frankfurt am Main : Fischer, 1969.

Horkheimer, Max, et Theodor W. Adorno. La dialectique de la raison. Paris: Gallimard, 1974.

Jay, Martin. The Dialectical Imagination: A History of the Frankfurt School and the Institute of Social Research, 1923-1950. $1^{\text {re }}$ éd. London : Heinemann, 1973.

Marramao, Giacomo. « Political Economy and Critical Theory ». Telos, nº 24 (1974) : 56-80.

Marx, Karl. Le Chapitre VI. Manuscrits de 1863-1867 - Le Capital, livre 1. Paris : Editions Sociales/Geme, 2010.

Neumann, Franz. Béhémoth. Structure et pratique du national-socialisme. Paris : Payot, 1987.

Pollock, Friedrich. « Is National Socialism a New Order? » Studies in Philosophy and Social Sciences IX (1941) : 440-55.

Pollock, Friedrich. «State Capitalism: Its Possibilities and Limitations ». In The Essential Frankfurt School Reader, édité par Andrew Arato et Eike Gebhardt, 71-94. New-York, NY : Continuum, 1982.

Postone, Moishe. Temps, travail et domination sociale. Paris : Fayard, 2009. 
Reijen, Willem van, et Jan Bransen. « The Disappearance of Class History in "Dialectic of Enlightenment" ». In Dialectic of Enlightenment, par Max Horkheimer et Theodor W. Adorno, 248-52. Stanford, CA : Stanford University Press, 2002.

\section{NOTES}

1. Max Horkheimer et Theodor W. Adorno, La dialectique de la raison, Paris, Gallimard, 1974, p. 129.

2. Cf. Martin Jay, The Dialectical Imagination: A History of the Frankfurt School and the Institute of Social Research, 1923-1950, $1^{\text {re }}$ éd., London, Heinemann, 1973; Barbara Brick et Moishe Postone, "Kririscher Pessimismus und die Grenzen des traditionellen Marxismus », dans Wolfgang Bonß et Axel Honneth (dir.), Sozialforschung als Kritik, Frankfurt am Main, Suhrkamp, 1982, p. 179-197; Moishe Postone, Temps, travail et domination sociale, Paris, Fayard, 2009.

3. Willem van Reijen et Jan Bransen, «The Disappearance of Class History in "Dialectic of Enlightenment" ", dans Max Horkheimer et Theodor W. Adorno, Dialectic of Enlightenment, Stanford, CA, Stanford University Press, 2002, p. 248-252.

4. Quoique les auteurs indiquent pourtant dans l'addendum à l'introduction de cette édition qu'aucune modification importante n'a été apportée au texte original, à part l'ajout du dernier fragment sur l'antisémitisme.

5. Willem van Reijen et Jan Bransen, op. cit., p. 251.

6. Rappelons tout de même que le gouvernement américain faisait surveiller quiconque était assimilé au «communism» par le House Committee of Un-American Activities, et ce dès avant le maccarthysme.

7. Cf. par ex. Martin Jay, op. cit., p. 165. Il est néanmoins à noter que l'exploration de Jay s'arrête en 1950, tandis que, notamment pour le cas d'Adorno, c'est bien après le mitan du siècle et le retour en Allemagne que se dessinent les traces éparses d'une réflexion sur l'économie politique. 8. Cf. à ce sujet Giacomo Marramao, "Political Economy and Critical Theory ", Telos, $n^{\circ} 24,1974$, p. 56-80. Figure quelque peu oubliée du marxisme, Henryk Grossmann a été l'un des rares auteurs à proposer une théorie de l'effondrement. Pour lui, en effet, le capitalisme tend inexorablement vers un point où la part de la survaleur que les capitalistes peuvent conserver pour leur consommation personnelle est réduite à néant, au profit de la part toujours croissante de survaleur destinée à la capitalisation et ce à cause notamment de l'importance toujours plus grande des machines et équipements technologiques dans les forces productives. Il arriverait donc un moment de crise finale où les capitalistes ne pourraient plus tirer aucun profit du système.

9. Friedrich Pollock, «State Capitalism: Its Possibilities and Limitations », dans Andrew Arato et Eike Gebhardt (dir.), The Essential Frankfurt School Reader, New-York, NY, Continuum, 1982, p. 72-3.

10. Friedrich Pollock, "Is National Socialism a New Order?", Studies in Philosophy and Social Sciences, vol. IX, 1941, p. 440-455.

11. Ibid., p. 453.

12. Friedrich Pollock, op. cit., p. 77.

13. Ibid., p. 87.

14. Ibid., p. 80.

15. Friedrich Pollock, loc. cit., p. 443.

16. Ibid.

17. Friedrich Pollock, op. cit., p. 78.

18. Friedrich Pollock, loc. cit., p. 449.

19. Franz Neumann, Béhémoth. Structure et pratique du national-socialisme, Paris, Payot, 1987, p. 215.

20. Ibid., p. 213. 
21. Ibid., p. 216.

22. Ibid., p. 333.

23. Ibid., p. 248.

24. Ibid., p. 250.

25. Ibid.

26. Ibid.

27. Ibid., p. 339.

28. Il existe néanmoins un texte de "Réflexions sur la théorie des classes » daté de 1942 et faisant une référence implicite à Neumann. Non publié du vivant d'Adorno. Nous y reviendrons ci-après.

29. Max Horkheimer, « L'État autoritaire », Théorie critique, Paris : Payot, 1978, p. 302.

30. Ibid., p. 303.

31. Ibid., p. 308.

32. Ibid.

33. Ibid., p. 322.

34. Katia Genel, Autorité et émancipation. Horkheimer et la Théorie critique, Paris, Payot, 2013, p. 253.

35. Max Horkheimer, « L'État autoritaire », op. cit., p. 307.

36. Ibid., p. 319.

37. Ibid., p. 303.

38. Ibid.

39. Max Horkheimer, Éclipse de la raison, Paris, Payot, 1974, p. 156.

40. Max Horkheimer, «L'État autoritaire », op. cit., p. 304.

41. Max Horkheimer, Éclipse de la raison, op. cit., p. 156.

42. Max Horkheimer et Theodor W. Adorno, La dialectique de la raison, op. cit., p. 169.

43. Appendice à la réédition de 1969 chez Fischer. Traduction anglaise, "The schema of mass culture ", parue in Theodor W. Adorno, The Culture Industry. Selected Essays on Mass Culture, New York, NY, Routledge, 1991.

44. Max Horkheimer et Theodor W. Adorno, La dialectique de la raison, op. cit., p. 174.

45. Ibid., p. 139.

46. Ibid., p. 169.

47. Ibid., p. 145.

48. Ibid., p. 146.

49. Ibid., p. 132.

50. Dans son essai «Sur la musique populaire », Adorno nomme «standardisation » et " pseudoindividualisation " les deux dimensions essentielles de cette schématisation. La première assure que chaque chanson à succès suive le schéma formel de toutes les chansons à succès; la seconde a pour but de "camoufler le prédigéré » c'est-à-dire de maquiller le caractère standardisé d'un tube au moyen d'effets et de gimmicks qui sont eux-mêmes répertoriables selon les fonctions limitées - pour lesquelles on peut les employer. «Par pseudo-individualisation nous entendons le fait de nimber la production culturelle de masse d'un halo de libre choix ou de marché libre sur la base de la standardisation elle-même. »

51. Max Horkheimer et Theodor W. Adorno, La dialectique de la raison, op. cit., p. 133.

52. Theodor W. Adorno, Minima Moralia. Réflexions sur la vie mutilée, Paris : Payot, 2003, p. 162.

53. Max Horkheimer et Theodor W. Adorno, La dialectique de la raison, op. cit., p. 132.

54. Ibid., p. 131.

55. Ibid., p. 168.

56. Cf. supra, note 50 .

57. Max Horkheimer et Theodor W. Adorno, La dialectique de la raison, op. cit., p. 163.

58. Ibid., p. 130.

59. Le terme lui-même apparaît dans la version allemande, Dialektik der Aufklärung mais la traduction en français lui a préféré l'expression peu claire d'« État de la prospérité » (p. 159). 
60. Max Horkheimer et Theodor W. Adorno, La dialectique de la raison, op. cit., p. 130.

61. Ibid., nous soulignons cette expression qui, comme indiqué en introduction du présent chapitre, remplace dans l'édition de 1947 celle de "monopole» qui était présente dans le tapuscrit de 1944, cf. Willem van Reijen et Jan Bransen, op. cit., p. 251.

62. Max Horkheimer et Theodor W. Adorno, "Das Schema der Massenkultur ", Dialektik der Aufklärung, Frankfurt am Main, Fischer, 1969.

63. Max Horkheimer et Theodor W. Adorno, La dialectique de la raison, op. cit., p. 148.

64. Ibid., p. 170.

65. Ibid. (*Nous avons modifié ici la traduction en revenant à l'original : l'édition en français indique « le fournisseur inconnu et le moins habile».)

66. Ibid., p. 171.

67. Ibid., p. 170.

68. Ibid., p. 133.

69. Theodor W. Adorno, "L'industrie culturelle », Communications, vol. 3, $\mathrm{n}^{\circ}$ 1, 1964, p. 13.

70. Ibid., p. 12.

71. Max Horkheimer et Theodor W. Adorno, La dialectique de la raison, op. cit., p. 131.

72. Ibid., p. 159.

73. Ibid., p. 152.

74. "Kein Hirt und eine Herde ", Max Horkheimer et Theodor W. Adorno, " Das Schema der Massenkultur ", op. cit.

75. Max Horkheimer et Theodor W. Adorno, La dialectique de la raison, op. cit., p. 175.

76. Dans son analyse du rapport entre Adorno et Pollock, Giacomo Marramao indique d'ailleurs qu'«Adorno a toujours considéré Grossmann comme le plus profond et le plus versatile des théoriciens de l'économie travaillant avec les catégories marxistes ». Cf. Marramao, loc. cit., p. 75.

77. Theodor W. Adorno, "Réflexions sur la théorie des classes», Société: IntégrationDésintégration. Écrits sociologiques, Paris, Payot, 2011, p. 65.

78. Ibid., p. 66.

79. Ibid., p. 63.

80. Ibid., p. 66.

81. Ibid., p. 76.

82. Voir note en introduction.

83. Theodor W. Adorno, "Réflexions sur la théorie des classes », op. cit., p. 77.

84. Dirk Braunstein s'est néanmoins essayé à cette systématisation. Cf. Adornos Kritik der politischen Ökonomie, Bielefeld, Transcript Verlag, 2014.

85. Theodor W. Adorno, Minima Moralia. Réflexions sur la vie mutilée, op. cit., p. 85. (* Nous avons ici choisi de revenir à la version originale. La traduction française indique "dans la société marchande », ce qui est moins précis quant au point qui nous intéresse ici.)

86. Ibid., p. 308.

87. Ibid., p. 305.

88. Ibid., p. 262.

89. D'après la transcription de ce séminaire paru sous le titre «Theodor W Adorno über Marx und die Grundbegriffe der soziologischen Theorie ", dans Hans-Georg Backhaus, Dialektik der Wertform, Fribourg, Ca ira, 1997, p. 501-513. Traduction en anglais dans la revue Historical Materialism, vol. 26, $\mathrm{n}^{\circ}$ 1, 2018 p. 154-164. Traduction partielle en français par David Buxton sous le titre «Sur Marx et les concepts de base de la théorie sociologique » dans la Web-revue des industries culturelles et numériques, juin 2018, assortie d'un commentaire du traducteur. Sauf indication contraire, nous nous reportons à cette traduction pour les extraits que nous citons ciaprès en français.

90. Ibid.

91. Ibid. 
92. Ibid.

93. Ibid.

94. Ibid.

95. Ibid.

96. Theodor W. Adorno, « L'industrie culturelle », loc. cit., p. 13.

97. Ibid.

98. Ibid., p. 14.

99. Ibid., p. 18.

100. Walter Benjamin, «L'ÆEuvre d'art à l'époque de sa reproductibilité technique (dernière version de 1939) », Euvres III, Paris, Gallimard, 2000, p. 269-316.

101. Theodor W. Adorno, « Société I », Société: Intégration-Désintégration. Écrits sociologiques, Paris, Payot, 2011, p. 33.

102. Theodor W. Adorno, "Capitalisme tardif ou société industrielle?», Société: IntégrationDésintégration. Écrits sociologiques, Paris, Payot, 2011, p. 85.

103. Ibid., p. 93.

104. Ibid.

105. Ibid., p. 86 .

106. Ibid., p. 87.

107. Ibid., p. 93.

108. Ibid.

109. Ibid., p. 92.

110. Ibid.

111. Karl Marx, Le Chapitre VI. Manuscrits de 1863-1867 - Le Capital, livre 1, Paris, Editions Sociales/ Geme, 2010, p. 182.

\section{RÉSUMÉS}

Cet article propose de reconstituer la genèse du concept d'industrie culturelle, employé par Max Horkheimer et Theodor W. Adorno dans leur ouvrage La dialectique de la raison puis repris par Adorno dans ses travaux ultérieurs. Nous cherchons à retracer l'émergence de ce concept, dans l'ensemble des réflexions critiques de l'Institut de recherches sociales de Francfort émigré à NewYork et notamment celles concernant la caractérisation du capitalisme d'après la Grande Dépression : entre capitalisme d'État et capitalisme des monopoles. Comme on le verra, il est en réalité impossible de dissocier l'industrie culturelle en tant que concept de ces réflexions plus générales sur la nouvelle forme de société inaugurée par le modèle soviétique, mais décelable à bien des égards dans le national-socialisme et jusque dans le capitalisme du compromis fordien.

\section{INDEX}

Mots-clés : Industrie culturelle, Theodor Adorno, Karl Marx, Capitalisme, Monopole 
AUTEUR

CHRISTOPHE MAGIS

Maître de conférences en Sciences de l'information et de la communication à l'Université Paris 8.

Membre du Centre d'Études sur les Médias, les Technologies et l'Internationalisation. 